\title{
„DIE OHNMACHT IST EIN SYNDROM VON DEM ÖSTERREICH.““ HEIMAT- UND SOZIALMISERE IN DEN STÜCKEN VON THOMAS ARZT
}

\begin{abstract}
Der Beitrag untersucht anhand zweier Stücke von Thomas Arzt (Die Neigung des Peter Rosegger, Totes Gebirge) literarische Darstellungsformen von Ansichten des gegenwärtigen Österreich. Der Fokus der Analyse richtet sich auf die poetisch originelle Art und Weise, in der der Dramatiker herkömmliche Motive wie Heimat, soziale Misere und österreichische Apokalypsestimmung präsentiert. Hierbei wird dargelegt, wie das transformative Spiel mit dramatischen Genres, Intertexten, signifikanten Idiomen und heimischem Liedgut eine Farce konstituiert, die aktuelle Verhältnisse und Mentalitäten transparent macht.
\end{abstract}

SChlÜsSELwÖRTER: Thomas Arzt, Soziales Drama, Sprachkritik, Farce, österreichische Befindlichkeit, soziale Kälte

\section{“POWERLESSNESS IS A SYNDROME OF AUSTRIA'S". HOMELAND AND SOCIAL MIS- ERY IN THE PLAYS OF THOMAS ARZT}

Abstract: With reference to two plays by Thomas Arzt (Die Neigung des Peter Rosegger, Totes Gebirge), this paper examines how attitudes in contemporary Austria are represented in literature. The analysis focuses on the playwright's poetical originality in presenting conventional themes like homeland, social misery and the Austrian sense of apocalypse. It will be argued that the transformative playing with dramatic genres, intertextuality, significant idioms and local song material is in fact a farce exposing current circumstances and mentalities.

KEYwORDS: Thomas Arzt, social Drama, criticism of language, farce, Austrian mood, social coldness

\section{Rissiger Sprachheimatboden}

Die postrealistischen Volksstücke von Thomas Arzt sind - wie die folgenden Ausführungen illustrieren sollen - vornehmlich von drei Themen geprägt: Heimat, Soziales und Sprache (vgl. Cerny 2011: 125), und alle drei erweisen sich genregemäß als

Günther A. Höfler - Universität Graz, Graz, guenther.hoefler@uni-graz.at 
ziemlich brüchig. Denn Heimat ist trotz der meist verzweifelten Versuche der Figuren letztlich weder geographisch noch sozial und auch nicht mental zu haben. Die Titel der bekanntesten Stücke des Schlierbacher Autors, also Grillenparz, Alpenvorland, Totes Gebirge signalisieren einen starken regionalen Heimatbezug, der sich allerdings als sehr prekär herausstellt. Und auch die Sprache ist nirgendwo beheimatet, sie ist durchwegs unnatürlich und mäandert zwischen Brachial- und Kunstdialekt, zwischen Sentenz und Absurdität, zwischen Fragment und Verstummen. Eine grundlegende sprachliche Labilität kennzeichnet generell die Rede der Figuren. Das Sprachbefremden schwingt in allen ihren Äußerungen mit, am deutlichsten formuliert dies der wiedergängerische Jäger in Grillenparz in einem schon tendenziell metadramatischen Monolog: „In gewisser Weise hat die Sprache mehr Menschen zerstört, als jedes andere Medium. Ist ein riesen Haufen Scheiße die Sprache" (Arzt 2011: 16). Und auch die Arbeiterin Flora im gleichen Stück hat Zweifel am Funktionieren der Sprache, die zumeist auf die Figuren so zugeschnitten ist wie ein entweder zu enges oder zu weites Gewand: „Kein Satz spricht aus, was getan wurde. Kein Wort verweist aufs Gefühlte. Wieso also sprechen?" (Arzt 2011: 26) Worte sind nicht bzw. nicht mehr in der Lage, Soziabilität aufrechtzuerhalten geschweige denn zu generieren. Die Rede ist letztlich von keinem Verständigungstelos getragen, sie führt die dramatis personae nicht zueinander, sondern entfernt sie immer weiter voneinander, was der Autor im Text oftmals durch markante Schweige-Markierungen kennzeichnet. Und diese Vereinzelungsdynamik zwischen den Figuren ist gewissermaßen ein Reflex des kollektiven Zustands, den der Psychiatrieinsasse Nepomuk (kein Zufallsname!) in Totes Gebirge folgendermaßen diagnostiziert: „Ein Ohnmachtssyndrom. Das Österreich [...] Ist in Dir. Das Österreich. Bis alles zerfällt." Der Mitpatient Raimund (auch sein Name fußt in der österreichischen Theatergeschichte) pflichtet ihm bei: „Es beginnt mit dem Versagen der Sprache“ (Arzt 2016b: 35f.). In diesem Stück ist die Fragmentierung der Verständigung sehr weit gediehen, so etwa bei einem Gespräch über den Wein:

Nepomuk: Nicht gut. Der beste. Nicht mal ein Glas. Wix ich. Das beste. Wix ich. Oder die ganze Flasche. Die. Raimund: Wir hätten so viel Besseres reden können, Finchen. Nepomuk: Die Flasche. Wix ich. Was für eine Flasche? Raimund: Es sind uns nur die Begriffe abhanden gekommen. (Arzt 2016b: 36)

Nun, ausgefeilte Dialogspannung würde sich anders ausnehmen als dieses elliptische aneinander Vorbeisprechen, das oft mit unerwarteten Repliken aufwartet, aber dennoch wenig Vorwärtspotential birgt. Die Rede tendiert zum Austausch von monologischen Äußerungen, und das heißt, dass sich im Grunde kaum Intrigenähnliches entwickelt. Und auch die Illusionsbildung ist folglich keine tragende Konstituente der Arztschen Dramaturgie. Es geht in diesen Dramen folglich nicht um Personalisierung von Lebensverhältnissen, wie noch im realistischen Sozialdrama etwa der 1970er Jahre, sondern ein durchgehend wahrnehmbarer auktorialer Zeigegestus stellt Zustände aus und führt häufig eine Reflexion soziologischer Erkenntnisse mit sich. Alles, was in den Heimat- 
stücken von Thomas Arzt gesprochen wird - und es wird viel gesprochen - steht also, selbstverständlich nur für die Figuren, auf sprachlich und kommunikativ unsicherem Boden, ähnlich der Rosegger-Statue in Die Neigung des Peter Rosegger, deren Fundament wiederholt nachgibt und die daher eine bedenkliche Neigung - natürlich nach rechts - aufweist.

\section{Der rechtslastige Rosegger: Eine Heimatfarce}

In diesem Stück, einem Auftragswerk des Grazer Schauspielhauses, ist das österreichische bzw. steirische Heimatbegehr am ausgeprägtesten dargestellt. Eine der diesbezüglichen Suchrichtungen formuliert der Gesangsverein, der hier den in fast allen Arzt-Stücken obligaten, kommentierenden Chor gibt: „Vagaunzoumdroum vomdochstoaau / Wouodlafliang und gamsalspringan [...] Undwoudezeit, deguadezeit [...] Nulebanduat untadeleit [...] Wou de knia nu in di stutzn / wou de bus $\mathrm{n}$ in da blus n“" (Arzt 2016a: 7 bzw. 37). Die Auslassungen der Chor-Varianten haben in den Arzt-Dramen selbstredend kaum die klassisch-räsonierende Funktion, sondern bringen vielfach - mehr oder minder leicht ironisch gebrochen durch das formale Arrangement den Volksgeist zum Ausdruck.

Die Pointe bei obiger Verballhornung der steirischen Landeshymne ist wohl, dass auf die Wo/wou-Eröffnung kein zu erwartender $\mathrm{Da}$ /do-Fingerzeig folgt, dass die quasiliturgische Heimatanbetung also ins Leere läuft und die Suche in unbestimmter Spannung verbleibt, es sei denn, man akzeptiert als Sehnsuchtsziel die Aussicht „Bisdwödunsam kravadlbockt“" (Arzt 2016a: 7). Fraglos drängt sich Peter Rosegger als Projektionsfläche für die Behandlung des Heimatthemas nahezu auf, die Feststellung der Archivarin Trost klingt demnach fast wie die Legitimation für die Verarbeitung von einigen originalen Textpassagen in diesem Stück: „Er hat [...] einen Boden geschaffen für eine ganze Vorstellung von dem, was Heimat sein könnte. [... Er] hat ja in seiner Zeit über eine alte Zeit geschrieben. Fast als könnt es auch ein Märchen sein“(Arzt 2016a: 29). Damit schlägt sie ihn nolens volens, obwohl sie sein Erbe kritisch hochhalten will, eindeutig der rückschrittlichen Seite zu, denn das „Glaubensbekenntnis des Reaktionärs“, so der Ideenhistoriker Mark Lilla, fokussiert auf einen ,glücklichen, wohlgeordneten Staat, in dem die Menschen ihren Platz kennen und in Harmonie zusammenleben, weil sie sich der Tradition und ihrem Gott unterwerfen." Mit dieser dichotomen propter hoc - post hoc-Narration erweise er sich als „Flüchtling der Geschichte“ (Lilla 2018: 20). Dass mit diesem Begriff wohl ein Gutteil der österreichischen Mentalität charakterisiert ist, sei nur am Rande angeführt.

Die Heimat im Rosegger-Stück ist auch deshalb brüchig, weil jeweils nach dem Absingen einer Heimatliedtravestie das Land von einer unerklärlichen Erschütterung erfasst wird, die die vermeintlich Heimat symbolisierende Dichterstatue jeweils in weitere Schieflage versetzt. Kurz zur verhandelten Geschichte, obwohl eine Story in 
den Stücken von Thomas Arzt nie dazu da ist, einen herkömmlichen Handlungsbogen aufzubauen mit entsprechender Spannungslösung, sondern eher als Rahmenhalt fungiert, der die einzelnen Szenen einmal mehr ein andermal weniger stringent zusammenhält. Von der Form her sind seine Dramen recht schillernd, sie bestehen zumeist aus einer Kombination von Elementen aus Volksstück, Stationendrama, Konversationsstück, Farce und Groteske. Sie sind ergo weder postdramatisch noch dramatisch-realistisch, sondern formale Hybride. Im konkreten Fall handelt es sich grosso modo um eine Far$\mathrm{ce}^{1}$, in der das Kippen der Statue in einem Provinzstädtchen Rätsel aufgibt. Und das ausgerechnet kurz, bevor eine Unesco-Kommission erwartet wird, die den Ort zum Weltkulturerbe erklären soll. Der aus Graz herbeigeholte Seismologe mit dem sinnigen Namen Heim mutmaßt eine Aktivität der eurasischen Platte, die die Steiermark zerreißen könnte, und ordnet Grabungen an, die die untergründigen Ursachen ans Tageslicht bringen sollen. Damit erfüllt er eine traditionelle Komödienformel, in der einem von außen Kommenden die Rolle zugedacht ist, Licht in verworrene Verhältnisse zu bringen. Wogegen sich der Unternehmer und glühende Rosegger-Fan Wiesinger beharrlich sträubt und sogar auf den Seismologen schießt (Arzt 2016a: 63), damit dieser seine Arbeit nicht erledigen kann. Der ebenfalls bedrohte Arbeiter Matthias liefert sodann die Pointe zum Geschehen, denn auf die Frage, was man denn sehen werde, antwortet er prompt: „Unsere Altlasten“ (Arzt 2016a: 55). Denn am Schluss stellt sich heraus, dass die Familie Wiesinger ihren Großvater, einen SS-ler, dort mit nach oben ausgestreckter Hand begraben hat, wo der bronzene Rosegger steht. Dieser kippt im Finale bei einem weiteren Beben auf Wiesinger, der aber im Epilog etwas versehrt mit einigem Grund auf gut österreichisch hoffen darf, dass er nicht vor Gericht kommt: „Das wird sich verlaufen. Ein lapidarer Provinzskandal“". (Arzt 2016a: 85) Er darf sogar hoffen, dass die zarte Liebe zur Archivleiterin Trost sich erfüllt, womit eine weitere Komödienanleihe bedient wäre. Doch die Handlung ist nicht das Primäre, es geht, so Martin Behr, um ,eine launige bis anarchische Erörterung des Heimatbegriffs, verwebt in aktuelle Gesellschaftspolitik mit germanistischen Erörterungen über die Ambivalenz des steirischen Heimatdichters" (Behr 2016). Wiesinger, der dringend wissen will, ob Rosegger wirklich ein Nazi war bzw. den Deutschnationalismus befördert hat, wartet mit markanten Zitaten auf, etwa: „Wenn es um Menschenrecht, Freiheit, Heimat und Volk geht, da wird Kanonendonner... zu einer magischen Musik“ (Arzt 2016a: 66). Und er will aus seiner Rosegger-Lektüre auch die Botschaft mitnehmen können, dass man Hilfe nicht bei den Ausländern verschwenden und sich stattdessen wieder um einen selbst kümmern solle (vgl. Arzt 2016a: 47). Frau Trost hingegen findet den Dichter bloß „Nostalgisch, kitschig und öd“, aber besonders in der Steiermark rede noch jeder von ihm, weil er "Gefühle erzeugt, Sehnsucht“ und, „weil man Angst hat, dass man sonst erkennt, dass da gar nichts ist, von einer Heimat" (Arzt 2016a: 32). An den beiden Rezeptionsweisen ist recht treffend die scheinbar beliebige Fungibilität des

\footnotetext{
${ }^{1}$ Es ist definitiv keine Groteske, wie Norbert Mayer behauptet. Vgl. Mayer (2016).
} 
Heimatdichters illustriert, die zumindest die steirische Auseinandersetzung mit ihm seit langem charakterisiert. Thomas Arzt lässt sehr markant mit Namen und ein wenig theatralischem Fleisch versehene mentale Positionen aufeinandertreffen; hierbei von einer Psychologie der Figuren zu sprechen wäre so gesehen ziemlich unangebracht. Dennoch entsteht durch das dramatische Arrangement tendenziell so etwas wie eine psychologische Größe, nämlich die Kollektivseele. Das funktioniert deshalb, weil der Zuschauer die den geäußerten Haltungen entsprechenden Affekte zu imaginieren veranlasst ist. Mit Unternehmer, Bürgermeisterin, Arbeiter, Wissenschaftler, Angestellter und Archivarin ist ein durchaus repräsentatives soziologisches Sample am Werk, das die heutige gesellschaftliche Befindlichkeit und die gefühlte Heimatlosigkeit veranschaulicht, die mit Roseggerschem Heimatsurrogat kompensiert wird. Und als Pendant dazu mit Fremdenaversion, wie sie etwa die Angestellte Elfriede zur Schau trägt, eine Verkörperung des neuen Prekariats, die ihre Haltung mit der typischen, abgestandenen Rhetorik vorträgt:

Je mehr Einwanderung, desto mehr will ich weg [...] Auf welchem Rücken wird das denn ausgetragen? [...] Schleichts euch, ihr Grenzproblem-Heruntermacher-und-Kompromiss-Scheiße-Finder [...] ihr Asylanten-Gutmenschen-und-Obergrenzen-Auslacher. (Arzt 2016a: 34 u. 67)

An dieser Figur zeigt der Autor sehr eindrucksvoll die Dynamik der Selbstaffektion, die die einschlägige Redeweise mit sich bringt bis hin zur völligen Verengung; gegen Schluss hin schwärmt sie gewaltaffin nur noch von „der Sache“ (Arzt 2016a: 70). Der Autor schaut dem Volk recht gewitzt aufs Maul und stellt sein Sprachverhalten nur leicht überspitzt aus. Da es sich um keine Charaktere im engeren Sinn handelt, kann auch kein Mitleid für sie aufkommen, sie können aber darum auch gewissermaßen nicht wirklich vorgeführt werden, darum hält sich auch selbst der komödientypische Verlacheffekt in Grenzen, abgesehen von manch situativer Sprachkomik, etwa, wenn die Bürgermeisterin vom Anfangsereignis erzählt: „Und dann seh ich, scheiße, das kulturelle Erbe hängt grad ein bisserl nach rechts“. (Arzt 2016a: 12) Die Personen stehen einfach für gegebene Haltungen, die ganz gut die These des Psychologen Drew Westen illustrieren, dass das politische Gehirn im Grunde ein emotionales Gehirn ist (vgl. Westen 2011: 44). Elfriede, die prototypisch zu kurz Gekommene, will auch, um ihren Opferstatus zu unterstreichen, von zwei Marokkanern angegrapscht worden sein; der auf diese Mitteilung folgende Dialog zwischen ihr und Matthias kann ohne Weiteres als repräsentativ für die „Verständigungs“ verhältnisse in Arzt-Dramen genommen angesehen werden:

Matthias: Wann ist das passiert? Elfriede: Was interessiert's dich jetzt? [...] Matthias: Das ist doch ein Scherz [...] Elfriede: Klar. Die Elfriede macht immer nur Scherze. Bis sich's aufhört. Aber dann versteh ich nix mehr. Matthias: Aber warum hast nix gesagt? Elfriede: Echt. Matthias: Was waren das für Marokkaner? Elfriede: Scheiße. Matthias: He. Elfriede. Elfriede: Aber hört ja keiner. (Arzt 2016a: 35) 
Eine ideologische Gegenposition zu Elfriedes Beschwörung einer autochthonen Identität mit den dazugehörigen Reinheitsphantasien nimmt die Bürgermeisterin als landläufige Stimme der Vernunft ein, die mit teils gestanztem Politvokabular den Grabenbruch verhindern möchte und Verständnis zeigt für alle Anliegen, die aber letztlich professionell auf Distanz bleibt, auch wenn sie in nahezu Schwabschen Sprachgebrauch das allgemeine „Beschissenheitsgefühl“ attackiert, also die „bevölkerungsverdichtende Angeschissenheitshaltung und eine Gesprächskultur vernichtende Scheiße-in-die-Öffentlichkeit-Herausschleuderungsbewegung" (Arzt 2016a: 35). Am Schluss eröffnet sie anstatt des Weltkulturerbes ein Landesseniorenheim. Der gerontisch-friedliche Ausgang des Stücks ist natürlich nur ein scheinbarer, denn im Grund bleiben alle Gräben offen. Unter der österreichischen Gemütlichkeit verbirgt sich hier wie in allen bekannten Antiheimat-Dramen reichlich Mulmiges, das nicht zuletzt mit dem in den Gschtanzeln zur Schau getragenen Zustandsbefund steirischer Männlichkeit zu tun haben mag: „Wou ma laff $\mathrm{n}$ duat und rauf a / Wou ma krax lt auf die wänd. / Wou ma schia $\beta \mathrm{n}$ duat und sauf a / Wou ma freind sand bis zum end [...] Wou ma dring an und daun speib m / und die gro ßn söh ne bleib m“ (Arzt 2016a: 35) - Andreas Gabalier lässt von gar nicht so fern grüßen. Der Dialekt, so der Autor, sei eine Urform, näher am Unbewussten, „da fallen die Masken“ (Mader 2016) - was zu zeigen war; die Sprachform verniedlicht die Brutalität und entbirgt sie zugleich. Thomas Arzt malt mit treffend gewählten Mitteln und Motiven ein Heimatbild, das seine sehr ambivalente Sicht zeigt auf ein „Österreich, das ich Heimat nenne und vor dem mir dabei immer wieder graust“ (Arzt, zit. nach Halter 2013). Es geht in seinen Stücken wohl auch darum, die Deutungshoheit über den Heimatbegriff nicht den Rechten zu überlassen, aber um diesen zu retten, ist es unabdingbar, in das Grausige zu blicken, das ihm unweigerlich anhaftet. „Ich weiß selbst nicht genau“, bekennt der Autor, „weshalb sich das Thema wie ein roter Faden durch meine Arbeit zieht [...] Mein jüngstes Stück [Totes Gebirge] ist gesellschaftspolitischer, bezieht sich auf die aktuelle Situation in Österreich" (Arzt, zit. nach Cerny 2016).

\section{Österreich - ein apokalypseträchtiges Eisgefängnis}

Schon die Perspektiven, die sich in diesem Stück zu Beginn des Geschehens auftun, das sich zwischen Weihnachten und Silvester in einer Psychiatrie abspielt, sind in keiner Weise rosig, der Puppenchor bringt es auf den Punkt: „Dahümmi feabalt sischwoaz / Dewoikn dunkin siei / [...] Dewöd stehtnimma nedlaung / Igspiascho denwöduntagaung“ (Arzt 2016b: 6). Die dunklen Wolken stehen für den Zustand der Gesellschaft, für die Herausbildung eines Eremitenklimas, das Alexander Mitscherlich schon Ende der 60er Jahre diagnostiziert (vgl. Bähr 2012: 162) hat und das sich im Zuge der neoliberalen Individualisierung enorm potenziert hat. Das Dunkle, das sind fraglos auch die Berge im Hintergrund mit ihrem klaustrischen Drohpotential - ein bewährtes, sym- 
bolträchtiges Motiv der österreichischen Literatur von Bernhard und Hüttenegger über Clemens Eich, Ransmayr bis jüngst zu Geiger, nämlich das Leben unter Einschluss durch Berge und jenseits resp. diesseits einer Grenze. Im vorliegenden Stück ist auch die Doppeltheit des Bildes durch die Puppen klar herausgestellt: „Kaweideslaund in unsrasö / Kaweideslaund inunsamhian [...] Nuadodebeag inunsrasö“ (Arzt 2016b: 53). Die Nachnamen der Figuren verdeutlichen die Innen-Außen-Entsprechung noch weiter, denn sie tragen die Namen von Gipfeln des toten Gebirges (Priel, Loser, Elm etc.), womit die Versteinerung in ihren Identitäten verankert wird. Auch die Ärztin sieht „eine bedrückende Ähnlichkeit. Von Land. Und Person. Eine Analogie“ (Arzt 2016b: 37). Das Schnitzlersche, Weite Land“ ist hier auf ein erodierendes Karstgebiet zusammengeschrumpft. Und auch der dramatische Rahmen unterstreicht die Erstarrung und die Kälte, die Akte sind betitelt mit „Schnee, Regen, Sturm, Frost, Eis“. Die psychiatrische Anstalt mit ihrem geringen Bewegungsraum reduziert die Aussichten im optischen und sozialen Sinn auf ein Minimum. Beziehungsweise auf Null, wie in der Perspektive des Nepomuk: „Hab alles gesehen. Das ganze Syndrom. Alles verdeppert in diesem Land [...] Kommt der Komet. Gegen das Land [...] Gegen das Syndrom. Gegen das Österreich“ (Arzt 2016b: 34f.). Die psychiatrische Anstalt ist bei Thomas Arzt keine Enklave, in der gemäß der antipsychiatrischen (Foucaultschen) Vision und deren Reflex in der Literatur der 1970er Jahre der Wahnsinn seine subversive Kraft entfalten kann, sondern sie ist ein Minimodell der Welt draußen. Die Welt als Irrenhaus oder Gefängnis, in dem Endzeitvisionen blühen, ist wohl auch kein ganz neues Motiv in der österreichischen Literatur. Die Insassen sind auf verschiedene, aber doch typische Weise im Leben gescheitert: Der Neuzugang Raimund, der anfangs sehr entrückt, nahezu kataton wirkt, hat sich nach einem Burn-Out selbst eingewiesen, er war Lehrer, ist „,verrückt" geworden und hat alle (natürlich!) Biedermeier-Möbel zerschlagen: „Ich hab nichts gemacht $[\ldots]$ Ich hab nur Literaturgeschichte unterrichtet $[\ldots]$ Ich war ein katastrophaler Lehrer in einem katastrophalen System" (Arzt 2016b: 66 u. 54). Aus dem Biedermeier sei er herausgewachsen, die gemütlichen Möbel in einer ungemütlichen Behausung seien ein Anachronismus, was wohl als Schnappschuss der österreichischen Mentalität rezipiert werden kann. Er wähnte sich als Schriftsteller vor dem Durchbruch, musste aber auf Druck des Vaters einen Brotberuf ergreifen. Nepomuk, der an einer alkoholinduzierten neuronalen Schwäche namens Marchiafava-Bignami-Syndrom leidet, beschwört manisch den zum Jahreswechsel erwarteten Kometen, und Emanuel war Langzeitarbeitsloser, lässt sich lethargisch treiben und ergeht sich in suizidalen Vorstellungen: „Irgendwann springt doch ein jeder vors Auto. Oder vor den Zug [...] Ich fahr in den Süden. Stell mich an eine Klippe. Und dann spring ich" (Arzt 2016b: 7 u. 64). Allenfalls noch rafft er sich etwas auf, um der Schwester von Raimund ein wenig den Hof zu machen.

Von einer aufbauenden Handlung kann auch in diesem Stück kaum die Rede sein, was das Geschehen anstößt, ist zum einen die Absicht der melancholischen Ärztin, den Hintergrund der Erkrankung der Patienten zu erkunden und zum anderen der Besuch 
Josefines, der Schwester Raimunds, die als Botin der Außenwelt als Stimme der Normalität fungiert und ein wenig Vorgeschichte einbringt, womit sie im Streit mit Raimund sogar für ein wenig Intrigenspannung sorgt. Im Allgemeinen werden die Leiden aber nicht durch Psychologisierung beglaubigt - das Empathievermögen des Zuschauers ist in Arzt-Stücken deutlich unterfordert - sondern im Zuge von Selbst- oder Fremdcharakterisierungen eher behauptet. Die Figuren lassen sich nicht aufeinander als Personen mit inneren Haltungen ein; Befindlichkeiten werden nicht gezeigt, sondern artikuliert, mitunter in poetischer Einkleidung: „Mein Herz ist wie erfroren“ - diesen Vers aus Wilhelm Müllers Winterreise hat Raimund hinterlassen (Arzt 2016b: 33), bevor er sich mit Pickel und Wanderschuhen wie der Protagonist aus dem besagten Poem aus dem erstarrten sozialen Klima der österreichischen Biedermeierlichkeit in die eisige Natur aufgemacht hat. Die sechs Akte sind, um die Unwirtlichkeit des Klimas zu betonen, betitelt mit „Schnee, Regen, Sturm, Frost, Eins, Schnee“. Im Gesang der vom Pfleger Anton verfertigten Puppen wird diese Seelenlage von Müller/Schubert-Niveau aufs Österreichische heruntergebrochen: „Meiheazal is dafroan / Meifreid hobi valoan / Isteh imschnee und schau / dabon brichtma davau“ (Arzt 2016b: 70). Der Chor mit seinem dialektalen Redegestus hat auch hier eine unglücksverkleinernde Funktion, diese sprachliche Verniedlichungsstrategie, die mitunter Volks- und Allerweltsweisheiten integriert, legt sich wie eine Decke auf das Geschehen, mit der Tendenz, die Spannungen zu ersticken. Haben doch die chorischen Einlagen laut dem Autor die Funktion, Konfliktvermeidungsstrategien aufzuzeigen (vgl. Hackl 2013). Dabei wird, etwa vom sozialkritischen Emanuel, das Grundproblem klar erfasst: „Es ist eine falsche Gesellschaft". Auf die pragmatische Replik der Ärztin hin, die Gesellschaft sei eben die Gesellschaft, insistiert er: „Aber wenn doch ein Riss da durchgeht“ (Arzt 2016b: 37). Hat Thomas Arzt in Alpenvorland (2013) noch die Mittelstandsverwahrlosung, die Heimatlosigkeit, die soziale Perspektiven- und Ortlosigkeit der Generation Y, also der 30plus-Kohorte zum Thema gemacht, ist hier der Blick auf das Ganze der Gesellschaft gerichtet, was in eine durchaus glaubhafte Inszenierung von Ausweglosigkeit mündet. Die Versehrtheit der Figuren ist eine Repräsentation des Sozialen, das in der (so Emanuel:) „Krankenmaschine“ (Arzt 2016b: 64) der Anstalt gespiegelt ist, die ebenso ein Kältetauschsystem ist wie die Gesellschaft und die winterliche Natur. Der Zufluchtsort Psychiatrie ist ein scheinbarer, er ist nämlich gleichermaßen ein Gefängnis, in dem die durch deregulierte Sozialverhältnisse bedingte personale Regression nicht mehr mit Euphemismen der Leistungsideologie übertüncht wird. Totes Gebirge ist gewissermaßen auch eine Art Gesellschaftsendspiel mit ironischem Ausgang: Den letzten Satz nämlich hat Nepomuk: „Sehen Sie. Frau Doktor. Der Komet“ (Arzt 2016b: 79). Die positive Alternative zum bad end formuliert der Puppenchor, allerdings im Irrealis: „Waundawaunsinnwiaraleachalschaß vafliagt [...] / Waundastüstaundausmlaundsiaußa schleicht [...] / Waundahoisobascheidastootsisöm dawiagt [...] / Jodaungangatsmavielleichtawengal guat" (Arzt 2016b: 39). Das Österreichische des Stücks besteht aber nicht nur in dieser unglückspflegerischen Antwort auf den Weltzustand, 
sondern auch in den einschlägigen literarischen und volkstümlichen Intertexten sowie in der sprachlichen Extravaganz zwischen Verspieltheit und Experiment. Denn: ein Weltuntergang ohne ein bisschen Gaudi wäre kein österreichischer.

\section{Literatur}

Arzt, Th. (2011). Grillenparz. Reinbek bei Hamburg: Rowohlt Theater Verlag.

Arzt, Th (2016a). Die Neigung des Peter Rosegger. Reinbek bei Hamburg: Rowohlt Theater Verlag.

Arzt, Th. (2016b). Totes Gebirge. Reinbek bei Hamburg: Rowohlt Theater Verlag.

Bähr, Chr. (2012). Der flexible Mensch auf der Bühne Sozialdramatik und Zeitdiagnose im Theater der Jahrtausendwende. Bielefeld: Transcript.

Behr, M. (17. September 2016). Starrer Heimatdichter blickt auf tiefe Gräben. Salzburger Nachrichten.

Cerny, K. (2011). Sommernachtsalbtraum. Profil, 42 (15).

Cerny, K. (2016). Wohin bewegt sich Europa, Herr Arzt? Profil, 47 (37).

Hackl, W. (22. April 2013). Chor der Identitätskrisen. Thomas Arzts „Alpenvorland“ im Linzer Landestheater. Der Standard.

Halter, M. (27. November 2013). Billy the Kid kam nur bis Deutschlandsberg. Frankfurter Allgemeine Zeitung.

Lilla, M. (2018). Der Glanz der Vergangenheit. Über den Geist der Reaktion (E. Liebl, Übers.). Zürich: NZZ Libro (Originalwerk veröffentlicht 2016).

Mader, B. (19. Januar 2016). Die Seele ist ein totes Gebirge. Thomas Arzt. Der Dramatiker über Normalität, Psychiatrie und die österreichische Kulturszene. Kurier.

Mayer, N. (17. September 2016). Peter Rosegger fällt nach rechts - oder links? Die Presse.

Westen, D. (2011). Das politische Gehirn (N. Hofmann, Übers.). Berlin: Suhrkamp (Originalwerk veröffentlicht 2007). 
